\title{
Video Article \\ At-Risk Butterfly Captive Propagation Programs to Enhance Life History Knowledge and Effective Ex Situ Conservation Techniques
}

\author{
Jaret C. Daniels ${ }^{1,2}$, Geena M. Hill ${ }^{2,3}$, Kristin A. Rossetti ${ }^{2}$, Stephanie J. Sanchez ${ }^{2}$, Jacob A. Hornfeldt ${ }^{1}$ \\ ${ }^{1}$ Department of Entomology and Nematology, University of Florida \\ ${ }^{2}$ Florida Museum of Natural History, University of Florida \\ ${ }^{3}$ School of Natural Resources and Environment, University of Florida
}

Correspondence to: Jaret C. Daniels at jdaniels@flmnh.ufl.edu

URL: https://www.jove.com/video/60591

DOI: doi:10.3791/60591

Keywords: Biology, Issue 156, butterfly, endangered species, Lycaenidae, conservation, captive propagation, rearing, life history, larvae, development time, luminous paint, exuviae

Date Published: 2/11/2020

Citation: Daniels, J.C., Hill, G.M., Rossetti, K.A., Sanchez, S.J., Hornfeldt, J.A. At-Risk Butterfly Captive Propagation Programs to Enhance Life History Knowledge and Effective Ex Situ Conservation Techniques. J. Vis. Exp. (156), e60591, doi:10.3791/60591 (2020).

\section{Abstract}

Improving knowledge of ex situ best practices for at-risk butterflies is important for generating successful conservation and recovery program outcomes. Research on such captive populations can also yield valuable data to address key information gaps about the behavior, life history, and ecology of the target taxa. We describe a protocol for captive propagation of the federally endangered Cyclargus thomasi bethunebakeri that can be used as a model for other at-risk butterfly ex situ programs, especially those in the family Lycaenidae. We further provide a simple and straightforward protocol for recording various life history metrics that can be useful for informing ex situ methodologies as well as adapted for laboratory studies of other lepidoptera.

\section{Video Link}

The video component of this article can be found at https://www.jove.com/video/60591/

\section{Introduction}

A growing list of studies indicates widespread and serious global declines in butterfly populations $s^{1,2,3,4,5}$. This includes the vast majority of atrisk species. Conservation programs designed to mitigate such declines often employ a mix of strategies including population monitoring, habitat management and restoration, scientific research, captive propagation, and organism translocation ${ }^{6}$. Within the U.S. and its territories alone, a total of 30 butterfly taxa are listed under the Endangered Species Act (ESA) as either threatened or endangered, with 21 of these having approved draft or final recovery plans. For such taxa, more than half of the identified recovery strategies recommend captive propagation or state that captive propagation should be assessed ${ }^{7}$. The use of ex situ conservation efforts for butterflies has grown considerably in recent years ${ }^{8,9}$, and has the potential to be a critical tool to aid recovery efforts ${ }^{10}$. Numerous institutions, organizations, and agencies are currently involved with ex situ efforts for at least 11 ESA-listed butterfly taxa (i.e., Cyclargus thomasi bethunebakeri, Euphydryas editha quino, Euphydryas editha taylori, Heraclides aristodemus, Hesperia dacotae, Lycaeides melissa samuelis, Oarisma poweshiek, Pyrgus ruralis lagunae, and Speryeria zerene hippolyta) and several other at-risk taxa (e.g., Callophrys irus, Euphydryas phaeton, Speyeria idalia, and Eumaeus atala) ${ }^{11}$ Despite the number of robust and successful efforts, there remains a lack of regular communication across programs and between conservation practitioners involving the exchange of ideas, data, effective methodologies, and results. Such knowledge sharing is essential as it helps minimize duplication of effort, improves overall best practices, and enhances conservation impact. Few published head-starting, captive rearing, breeding, or husbandry protocols are readily available for at-risk butterfly taxa, and those that are often lack sufficient narrative detail and/or illustrations. These often provide mostly summary details with limited step-by-step instructions and accompanying images, making replication challenging or application to other taxa difficult to assess ${ }^{12,13,14,15}$. Many of the available protocols are limited in some way: they exist only in the gray literature, or in varying levels of detail, age of publication, or as component parts in symposium proceedings, agency/funder reports, or inhouse manuals $16,17,18,19,20,21,22,23,24$.

For most conservation programs, captive propagation is primarily conducted to support conservation translocation, which encompasses reintroduction, reinforcement (i.e., augmentation), and introduction ${ }^{25,26}$. Such activities are intended to be implemented strategically as a component of the overall recovery strategy in order to help prevent the extinction of a listed species, subspecies, or populations. It should be noted, however, that this is one of several other potential roles that such ex situ programs may serve. These may also include maintaining an insurance (i.e., refugia) population, temporary organism rescue, supporting recovery-related research and/or training, and promoting conservation-related education and awareness efforts ${ }^{27,28}$. Regardless of whether ex situ programs have a single defined goal or a mix of several, conservation practitioners should maximize opportunities for data collection in order to fill in key information gaps when possible. This is particularly important because the vast majority of at-risk taxa have generally been poorly studied prior to substantial wild population declines. The resulting enhanced knowledge gained on various behavioral, ecological, or life history aspects of the focal taxon can serve to help advance effective species conservation and management ${ }^{29}$. 
Here, we describe in detail the captive propagation protocol that was developed for the federally endangered Miami blue butterfly (Cyclargus thomasi bethunebakeri) (Supplementary Figure 1) as part of a larger conservation and recovery program. In this case, the captive propagation program serves three specific identified roles: 1) an insurance population should the existing wild population be lost, 2) a research population designed to fill in identified ecological and life history knowledge gaps that may help inform recovery and/or management, and 3 ) to produce viable organisms for conservation translocation into sites within the taxon's historical range. The resulting protocol has been well vetted and proven, having been utilized and improved for over a decade. Consequently, we feel that the described techniques and methodologies represent a viable model that can be applied to or readily adapted for other ex situ at-risk butterfly programs, particularly those involving Lycaenidae or related taxa. While we do not suggest that the described protocol is superior to others, we feel that there are opportunities for applying some of the methods more broadly to help enhance productivity, care, or efficiency. This is particularly true as much of our breeding is done under indoor laboratory conditions with limited space, similar to the conservation programs involving Euphydryas editha taylori and Speryeria zerene hippolyta ${ }^{17,23}$. Numerous other protocols often utilize potted material for oviposition or larval rearing, which can sometimes lead to increased complexities related to predator control, environmental control (i.e., humidity, temperature), livestock monitoring, data collection, plant pest issues, and space to name a few ${ }^{21,22}$. Lastly, the presented protocol outlines the methods for captive breeding. Many other at-risk butterfly conservation programs involve head-starting or captive rearing with the representative protocols reflecting those differences. While often minor, we feel that this helps broaden the existing pool of available information for other programs to review. This is critical, because most ex situ programs represent pioneering efforts to help facilitate the recovery of rare and often poorly studied taxa. Available protocols can serve as an excellent starting point to help provide valuable insight, reduce duplication of effort, and promote innovation. Owing to "the extensive interspecific diversity of butterfly behaviors, life history traits, and ecological requirements combined with often marked differences in program facilities, budgets, practitioner expertise" and other inherent differences, reliance to a single methodology, even for closely related taxa, is often limiting and unwarranted ${ }^{30}$. Flexibility to refine or develop new protocols tailored to the needs of specific taxa or programs is essential for success and should therefore be emphasized. We additionally describe laboratory techniques for collecting metrics on organism development under captive conditions including the number of larval instars, duration of individual developmental stages, total development time, and larval and pupal length. These techniques have broad applicability for life history studies of Lepidoptera that can be used to refine ex situ protocols or inform field data.

\section{Securing successful adult courtship and mating}

1. Following successful eclosion, release viable adult butterflies into a secure, walk-in, screened flight cage located in a temperature-controlled greenhouse (Supplementary Figure 2).

NOTE: Adults can be marked on the ventral surface of the wings with permanent ink markers if identification of specific individuals is desired for separation of genetic lines, stock origin, or for specific data collection related to organism longevity, behavior, etc.

1. While the exact cage dimensions can vary, ensure that there is ample space to accommodate adequate nectar plant material necessary to support the density of housed adult butterflies and provide room for a human to freely stand and pivot around.

2. Beyond temperature regulation, ensure that the greenhouse is secure so that it can provide a second layer of containment along with protection from inclement weather (e.g., heavy rain, wind).

2. Elevate potted nectar plant material so that there is no more than $30 \mathrm{~cm}$ of space from the interior top of the cage to the highest blooming flowers (Supplementary Figure 2). This provides optimal access to the available nectar resources, offers ample adult perches, and minimizes extraneous flight activity.

3. Place one potted host plant in the flight cage. This ensures that even if a mated pair is missed any resulting eggs laid can be collected.

4. Provide consistent airflow. This enhances courtship activity and mating success. In a greenhouse setting, blowers and fixed mount circulation fans are best used to help enhance ventilation and air movement. Smaller portable ventilation, such as box or desk fans, can also be used.

5. Maintain the internal greenhouse temperature between $27^{\circ} \mathrm{C}$ and $32^{\circ} \mathrm{C}$ to promote optimal adult activity and mating success. The temperature inside the cage is monitored using a traceable memory monitoring thermometer.

6. Mist the screened flight cage regularly (approximately once every $2 \mathrm{~h}$ ) with water using a hand pump, plastic tank sprayer, or garden hose.

7. Gently collect individual mating pairs from the screened flight cage using a 50 dram clear plastic snap cap vial (Table of Materials), placing one to two pairs per vial, and transport to an indoor rearing room or laboratory (Supplementary Figure 3).

\section{Maximizing egg production}

1. Assemble oviposition chamber.

1. Take a 12 ounce plain white paper cup and using a snap blade utility knife, make two horizontal cuts on each side of the cup across from one another. Each cut should be approximately $1 \mathrm{~cm}$ below the rim.

2. Cut a single cotton swab in half and insert the rod end of each into the two horizontal cuts on each side of the paper cup so that the cotton swab portion extends approximately $2 \mathrm{~cm}$ towards the interior of the cup.

3. Using a snap blade utility knife, make two "X" cuts in the bottom of the paper cup. Each diagonal cut should be approximately $1 \mathrm{~cm}$ long.

4. Take a 9 ounce plastic cup and fill the bottom with approximately $2 \mathrm{~cm}$ of tap water.

5. Place a fresh cutting, approximately $15 \mathrm{~cm}$ long, of terminal larval host plant growth in the paper cup by inserting the stem through one of the " $X$ " cuts in the bottom. Push the stem through the cut so that approximately $4-5 \mathrm{~cm}$ protrudes out the bottom.

6. Place the paper cup with host material into the plastic cup, ensuring that the plant stem is in water.

7. Fill a $1 \mathrm{ml}$ sub-Q syringe $(0.45 \mathrm{~mm} \times 16 \mathrm{~mm})$ with a flavored sports drink and saturate both cotton swabs in the paper cup. These act as artificial flowers.

NOTE: Melon and fruit punch flavored sports drink provide the best nectar alternative.

8. Once each mating pair separates, place 2-3 gravid females into the assembled cup configuration (i.e., the oviposition chamber). 
9. Cover the cup with a cut square fragment of black tulle (approximately $15 \mathrm{~cm} \times 15 \mathrm{~cm}$ ) and secure it with a rubber band around the lid (Supplementary Figure 4). Black tulle provides the best visibility into the cup and easy identification of any eggs that might occasionally be laid on the tulle.

2. Stimulate adult butterfly activity and oviposition.

1. Place each oviposition chamber on a laboratory bench or table approximately $19 \mathrm{~cm}$ below an $8.5 \mathrm{inch}(21.59 \mathrm{~cm}) \mathrm{clamp} \mathrm{light} \mathrm{with} \mathrm{an}$ aluminum reflector housing a $40 \mathrm{~W}$ incandescent bulb (Supplementary Figure 5).

NOTE: The incandescent light provides the radiant heat necessary to stimulate adult activity and oviposition.

2. Place a traceable memory monitoring thermometer adjacent to the lights and run the temperature sensor so that it rests on top of an oviposition chamber located directly under a clamp light.

NOTE: The target temperature range is between $27.5^{\circ} \mathrm{C}-29^{\circ} \mathrm{C}$.

3. Add supplemental clamp lights as necessary depending on the total number of ovipositional chambers deployed.

4. Plug clamp lights into a programmable $15 \mathrm{Amp} 24 \mathrm{~h}$ indoor plug-in mechanical timer with two outlets (programmable in $30 \mathrm{~min}$ timed intervals).

5. Set timer to turn clamp light on for $\mathbf{3 0}$ min intervals (i.e., a repeatable cycle of $\mathbf{3 0} \mathrm{min}$ on, $30 \mathrm{~min}$ off).

NOTE: This light cycle helps to maximize egg production by providing repeatable periods of illumination to stimulate adult butterfly activity and oviposition followed by short dark rest periods.

6. Refresh the cotton swabs in each cup with flavored sports drink via the sub-Q syringe and mist regularly with water using a plastic spray bottle approximately every 2-3 $\mathrm{h}$ or as needed.

NOTE: This provides adequate artificial nectar and moisture to enable the butterflies to free-feed as desired. It thereby enhances both adult longevity and oviposition productivity under laboratory conditions where living, blooming plant material cannot easily be utilized.

7. Monitor cups regularly and replace host plant with fresh cuttings as necessary.

8. When the eggs start to hatch or the density of eggs becomes high, move the female(s) to a new cup with fresh host and begin larvae protocol with neonates.

\section{Larval care and maintenance}

1. Repeat steps $2.3-2.6$ to assemble cups for larvae.

2. When eggs begin to hatch, move host plant material with eggs and neonate larvae into a newly assembled cup, placing the stem through the second " $X$ " in the bottom ensuring that the plant stem is in water and leaves touch adjacent fresh host cutting.

3. When larvae are young (neonate-2 instar), check larval cups daily for freshness of host plant material and presence of mold or excessive frass.

NOTE: Daily removal of host material is not recommended when larvae are young because this can result in organism injury due to handling and/or unnecessary waste of fresh host material.

4. If host material is wilted or in otherwise poor condition, place another cutting of fresh host material into cup so that it is touching existing foliage and allow larvae to move to the new host on their own.

5. Once larvae reach the $3^{\text {rd }}$ instar, replace paper cup and add fresh host material daily.

6. Use a small camel hair watercolor paint brush to gently move the larvae from the old host material or cup surface to fresh host material in the new cup.

7. Place the old host material into an empty rectangular plastic food storage container.

8. Repeat steps 3.5-3.7 daily and until all cups with larvae have been processed.

9. When complete, add a small amount of fresh host material on top of the plant waste in the food storage container and loosely place a lid on top.

NOTE: This serves as a safeguard in case any larvae are overlooked during daily processing because they will crawl onto the new host material on top of the plant waste and can be removed the next day.

10. Maintain cups under laboratory temperature between $25^{\circ} \mathrm{C}-28^{\circ} \mathrm{C}$ for optimal larval activity and development (Supplementary Figure 6). NOTE: To reach optimal rearing temperatures under indoor conditions, it is often necessary to place cups under clamp lights with aluminum reflectors housing $40 \mathrm{~W}$ incandescent bulbs. Temperatures can then be actively monitored using a traceable memory monitoring thermometer and the light height adjusted to reach optimal rearing conditions.

\section{Constructing the pupation chamber}

1. Cut a single face corrugated paper roll into equal sized $3.8 \mathrm{~cm} \times 3.8 \mathrm{~cm}$ squares.

2. Place one square into a 2 ounce clear plastic portion cup.

3. Place the cup onto a clear plastic cup tray (Supplementary Figure 7).

\section{Preparing larvae for pupation}

1. Identify mature larvae ready to pupate during daily colony processing.

NOTE: Such larvae will turn a uniform dull greenish-brown, lose their chevrons, and often wander off the host.

2. Gently remove each mature larva with a small camel hair watercolor paint brush or forceps and place one in each pupation chamber.

3. Firmly snap the clear plastic lid on the pupation chamber.

4. Repeat steps 5.1-5.3 until all larvae ready to pupate have been transferred into pupation chambers adding new plastic trays as necessary (Supplementary Figure 8). 


\section{Maintaining pupae}

1. For each tray of pupation chambers, record the date of first pupation and any other pertinent information needed (i.e., genetic line, experimental trial, etc.).

2. Organize trays by date and place in a secure location within the laboratory (Supplementary Figure 8).

3. Monitor trays daily for adult eclosion.

NOTE: Laboratory conditions such as temperature will strongly influence development time.

4. Prior to adult eclosion (typically within 10 days of first pupation), remove the lids from individual pupation chambers and place the tray in a $34.29 \mathrm{~cm} \times 34.29 \mathrm{~cm} \times 60.96 \mathrm{~cm}$ collapsible mesh pop-up rearing cage (Supplementary Figure 9).

NOTE: Pupae securely attached within the grooves of the corrugated paper squares facilitate successful adult eclosion (Supplementary Figure 10).

5. Repeat the entire protocol from step 1.1 for the subsequent captive generation.

\section{Assessing development time of immature stages and the number of stadia}

1. Place a single larva under a dissecting microscope. Use a small camel hair watercolor paint brush to carefully move and isolate larvae to avoid injury to the organism.

2. Dip a single hair of the paintbrush in nontoxic luminous paint (Table of Materials), and carefully put one small drop of paint on the back (dorsum) of the larva. Use a paint color that stands out from the background color and pattern color of the larva (Supplementary Figure 11). Be sure to avoid placing paint on the head of the larva.

3. Once the paint dries (about $30 \mathrm{~s}$ or so), place each individual larva in its own 2 ounce clear plastic portion cup containing approximately 1-3 small leaves of fresh terminal host material and write a unique identifier on the cup and lid (Supplementary Figure 12).

4. Carefully check each larva daily. Remove leaves and set onto white surface. Inspect cup, clear lid, and examine leaves under a dissecting microscope for the presence of larval exuviae (cast skins) and/or head capsules.

5. If a larval exuvia is found, remove it from the cup and place it in a microcentrifuge tube labelled with the corresponding cup number and the date (see steps 8.1-8.6. below).

6. Repaint larvae after each molt and record molt dates.

7. Measure the total body length (head to last abdominal segment) of each larva daily using digital calipers. Take three measurements and record the average of the three, along with the date and time. For early instar larvae, a magnifying glass or dissecting scope should be used when measuring to ensure accurate measurements.

8. Return larva to its corresponding plastic portion cup.

9. Add fresh host material as needed and remove all frass and old host debris. If mold is found in the cup, dispose and use a new cup. Write the correct unique identifier number on the new cup.

10. Repeat steps 7.5-7.9 until all larvae reach their final instar and begin the prepupal stage. When larvae cease feeding, turn a uniform dull greenish-brown color, lose their chevrons, and often wander off the host, minimize disturbing them.

11. Place a small piece of corrugated paper in the cup (see step 4.1).

12. Once each larva has fully pupated, measure its total length as in step 7.8 above and record the date of pupation. This will be the final molt of each individual.

13. Check on pupae daily and record eclosion date and sex of each resulting adult butterfly.

14. Measure the wing chord length of each butterfly using digital calipers. Butterflies can be gently held with forceps for measurement. If the butterfly is too active to easily measure, temporarily place it in a refrigerator for $30 \mathrm{~s}$ or less and try again.

\section{Collecting larval exuviae}

1. When a larval exuvia is observed, fill a microcentrifuge tube with $0.2 \mu \mathrm{l}$ of glycerin. Label the top of the lid and the side with the larva number, date of molt, and head capsule (H.C.)

NOTE: The larvae of some lepidopteran larvae regularly consume their exuviae but the head capsule should remain.

2. Place the larval exuvia and the associated head capsule in a clear plastic portion cup lid and put a couple of drops of ethanol in it

3. Examine the larval exuvia under a dissecting microscope by placing it in a clear plastic portion cup lid and putting a few drops of ethanol on it. If the larval head capsule is already separated from the exuvia, place a drop of glycerin on the tip of pointed entomological forceps and gently touch the head capsule to the glycerin. Place the head capsule in the associated microcentrifuge tube.

4. If the head capsule is still attached to the larval exuvia, use pointed forceps and an insect pin to separate the head capsule from the larval exuvia.

5. Once it is separated, use the glycerin technique to pick up the head capsule. If there is too much ethanol, you can use a small paper towel to remove some, but be careful not to accidentally remove the head capsule.

6. Place the head capsule in a labelled glycerin-filled vial and close lid tightly. 


\section{Representative Results}

Over the course of two separate conservation initiatives targeting the recovery of Cyclargus thomasi bethunebakeri from February 2003 to December 2010 and from November 2016 to the present, this protocol was used to successfully produce an excess of 51,052 viable organisms. Based on the one-year summary snapshot of overall captive population productivity from June 2018 to June 2019 , a total of 10,166 viable organisms were produced, representing $782.00 \pm 118.93$ organisms per month over 13 generations. Similarly, mean total egg production per female under laboratory conditions was $114.00 \pm 26.12(n=12)^{31}$. The resulting substantial organism productivity ranks this program among the largest such ex situ efforts in the U.S., along with those of Euphydryas editha taylori, Speyeria zerene hippolyta, and Lycaeides melissa samuelis ${ }^{24}$. Part of this productivity can be attributed to the fact that the butterfly is continuously brooded, producing one generation approximately every 4-6 weeks in captivity. The majority of other conservation breeding programs involve taxa that are univoltine or bivoltine. Nonetheless, even for programs involving extremely fecund taxa such as Speyeria spp., the total number of viable organisms produced for conservation translocation on an annual basis seldom exceeds a few thousand ${ }^{32}$. Accordingly, our captive population has enabled directed research and extensive data collection on numerous key data gaps important to improve best laboratory breeding and husbandry practices (Figure 1) as well as help inform recovery and management decisions.

Mean total development time from neonate larva to adult was 28.63 days (Table 1). The majority of larvae had four molts (Figure 2, Figure 3), though two had five molts, and one had six molts. The overall mean length of all larval instars was $5.97 \mathrm{~mm}$, and larvae were largest at the fourth and prepupal life stages (Table 1). When only including variables with more than 30 observations, the shortest time was spent in the first instar and prepupal stages, and the longest was spent as pupae (Table 1, Figure 2). Females typically developed quicker in all immature stages compared to males, although this was not a significant effect $(p=0.625)$. Statistical analyses were conducted using RStudio Version $1.1 .463(R$ Core Team 2016) ${ }^{33}$. The mean adult wing chord length was $12.64 \mathrm{~mm}$ (Table 2), and there was a significant difference between the sexes ( $p$ $=0.047$ ). The two-sided t-test was run to evaluate the wing chord difference between sexes. Linear regression model and stepwise regression for average length of each life stage showed that pupal length was the best predictor for adult wing chord length (Table 3, Table 4). Regression models for development time showed that the number of days spent in the second and fourth instars and the total number of days were the best predictors for adult wing chord length, but only the number of days in fourth instar was significant (Table 5, Table 6). Because variables were continuous, two linear regression models were run for the development time of each life stage, as well as the length of each life stage, with adult wing chord length as the dependent variable. Stepwise regressions were run on both regression models to determine the best predictors of adult wing chord length.

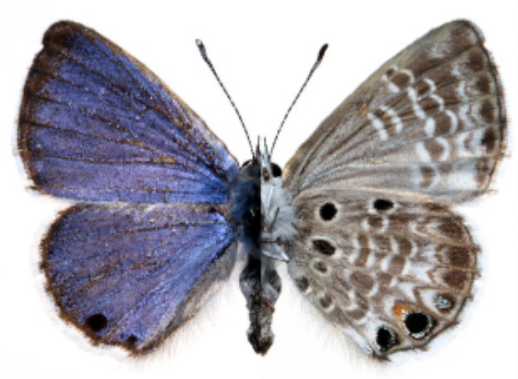

A

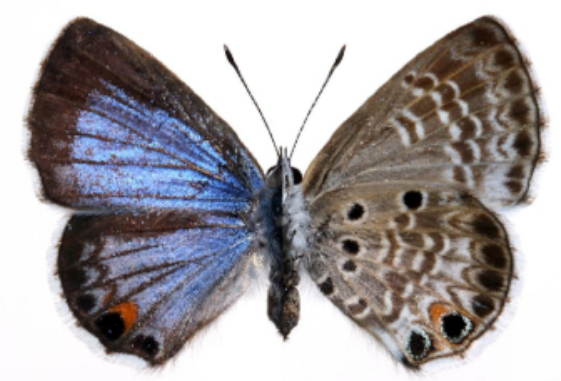

B

Supplementary Figure 1: Pinned specimens of adult Cyclargus thomasi bethunebackeri. (A) Adult male, dorsal (left), ventral (right). (B) Adult female, dorsal (left), ventral (right). Please click here to view a larger version of this figure. 


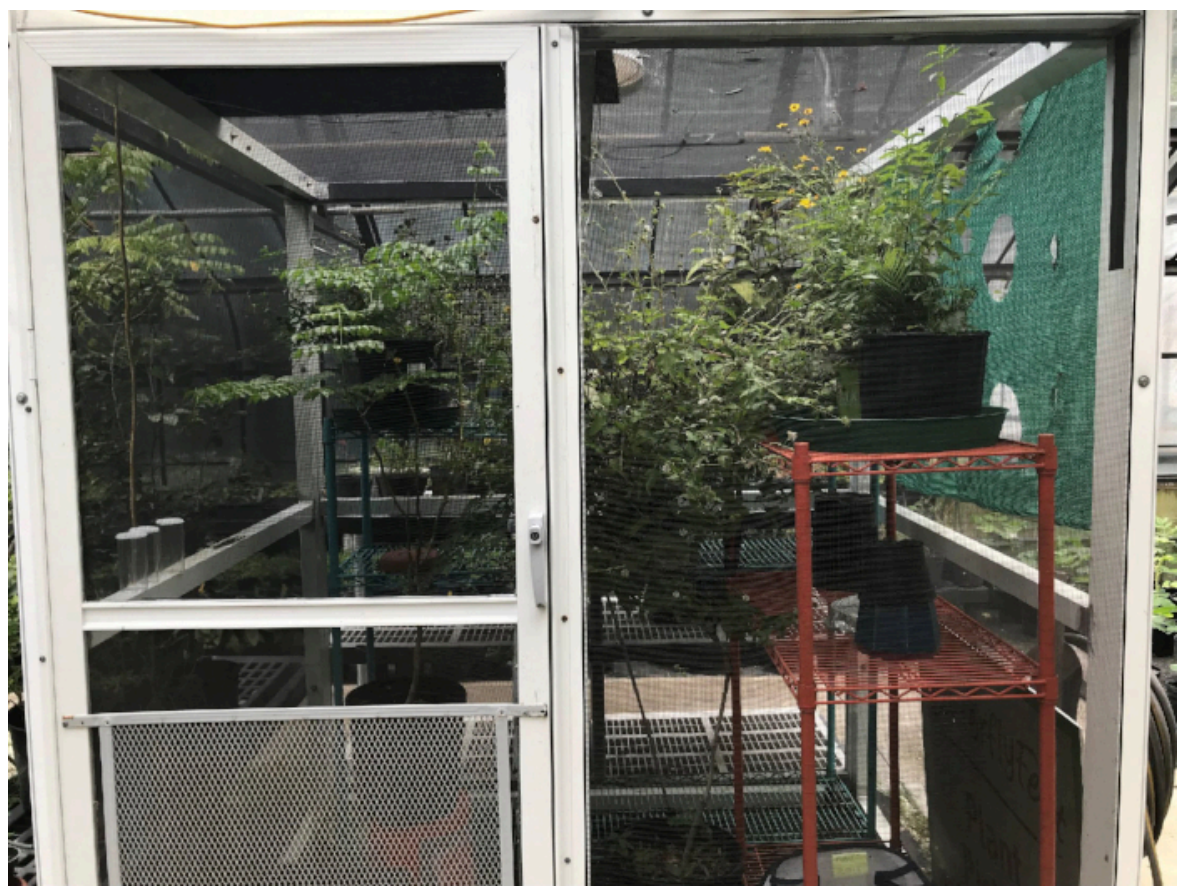

Supplementary Figure 2: Screened flight cage housed in temperature-controlled greenhouse. (A) Interior shows potted adult nectar plants and a single potted larval host plant. (B) Metal shelving helps to elevate potted nectar plants so that there is no more than $30 \mathrm{~cm}$ of space from the interior top of the cage to the highest blooming flowers. Please click here to view a larger version of this figure.

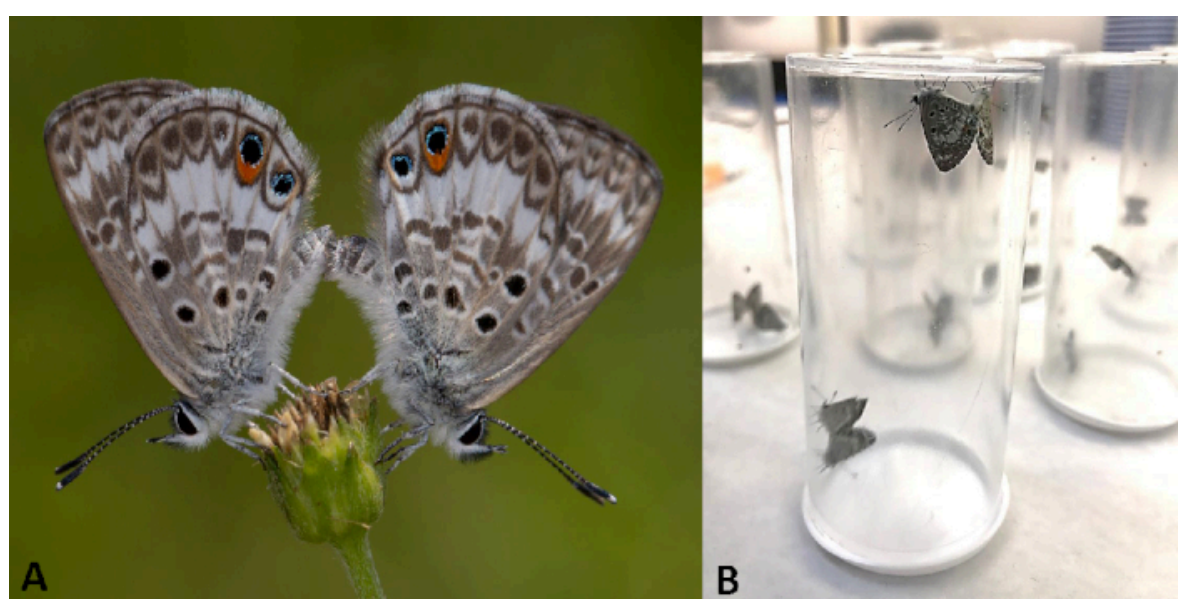

Supplementary Figure 3: Procedure for collecting adult pairs in copula. (A) Mating pair of adult Cyclargus thomasi bethunebakeri inside the screened flight cage (female, right and male, left). (B) Mating pairs collected from the flight cage in snap cap vials and brought into the laboratory. Please click here to view a larger version of this figure. 


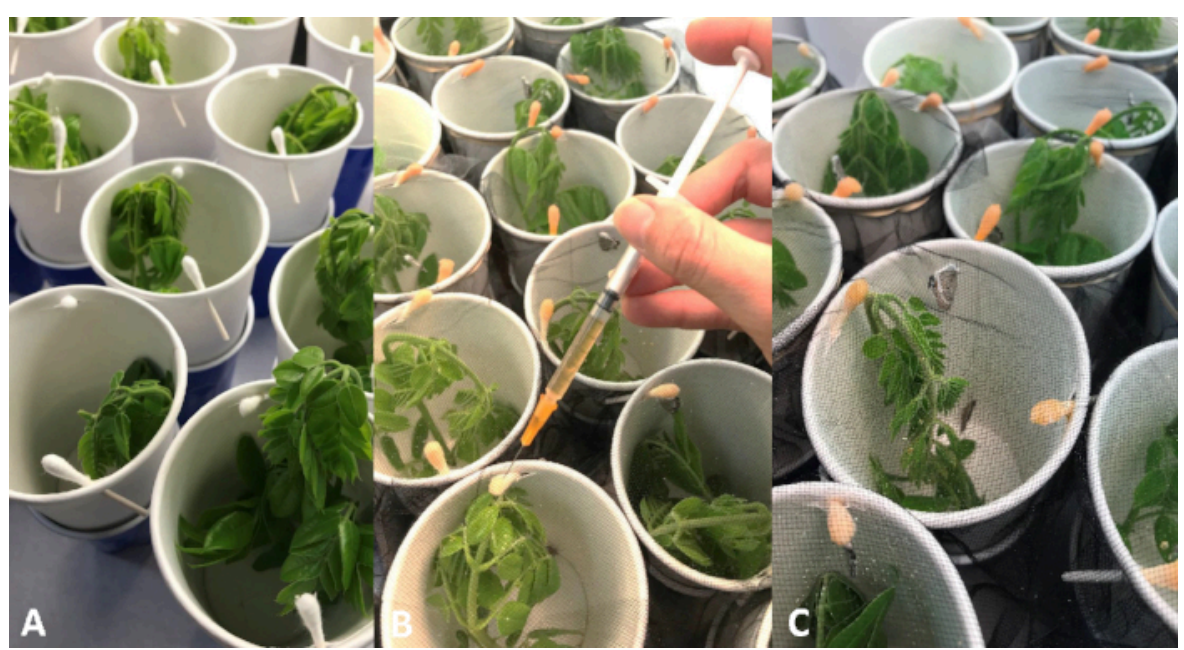

Supplementary Figure 4: Procedure for assembling oviposition chamber. (A) Two cup system with terminal host material and cotton swabs. (B) A $1 \mathrm{ml}$ sub-Q syringe $(0.45 \mathrm{~mm} \times 16 \mathrm{~mm})$ with flavored sports drink saturating cotton swabs in the paper cup. (C) Cups housing gravid females secured with black tulle. Please click here to view a larger version of this figure. 


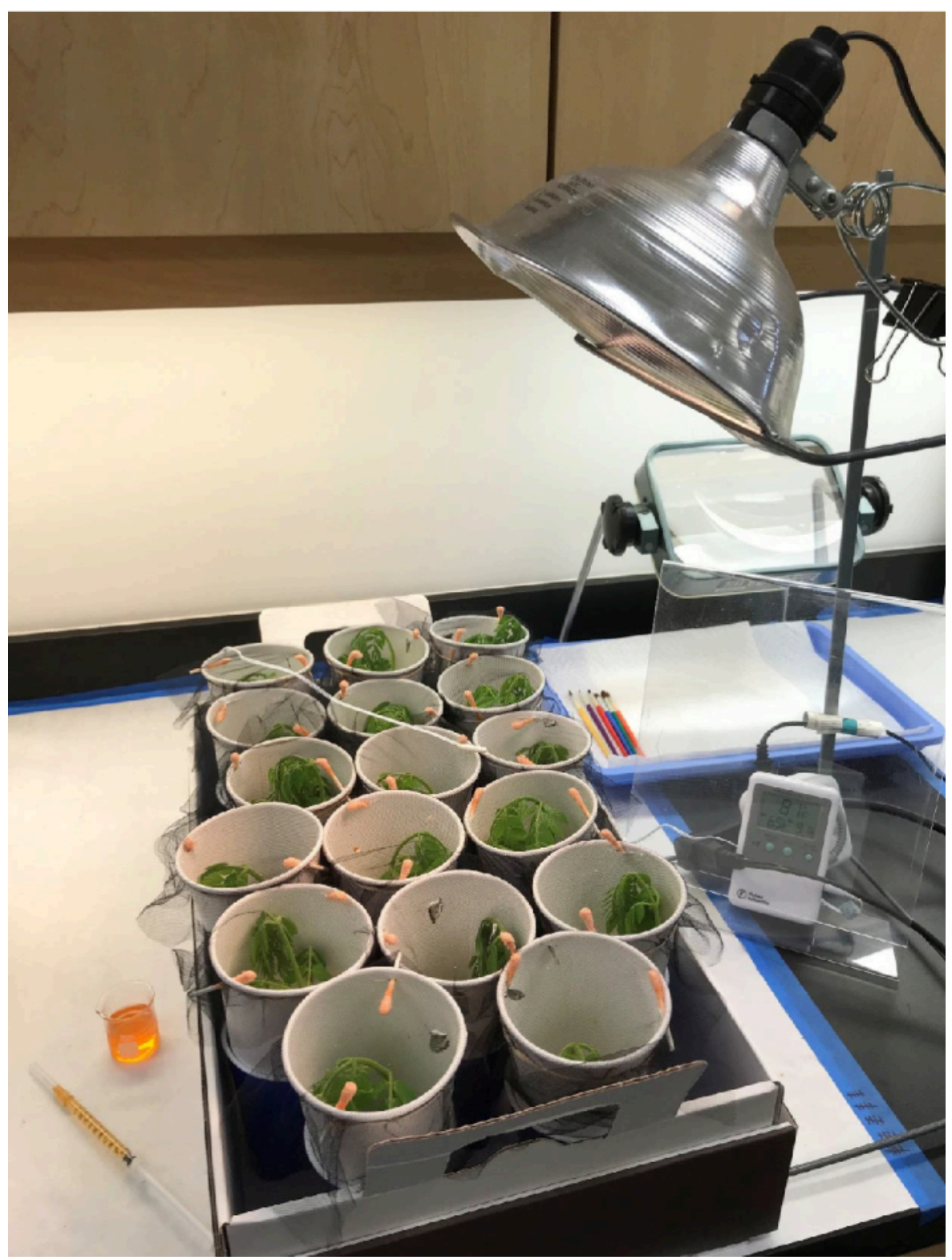

Supplementary Figure 5: Laboratory setup for maximizing egg production. (A) Oviposition chambers placed on a laboratory bench under a clamp light with a $40 \mathrm{~W}$ incandescent bulb. (B) A traceable memory monitoring thermometer is placed adjacent to the lights with the temperature sensor resting on top of an oviposition chamber located directly under a clamp light. (C) A $1 \mathrm{ml}$ sub-Q syringe and small beaker holding flavored sports drink placed adjacent to the oviposition chambers to facilitate refreshing the cotton swabs regularly throughout the day. Please click here to view a larger version of this figure. 


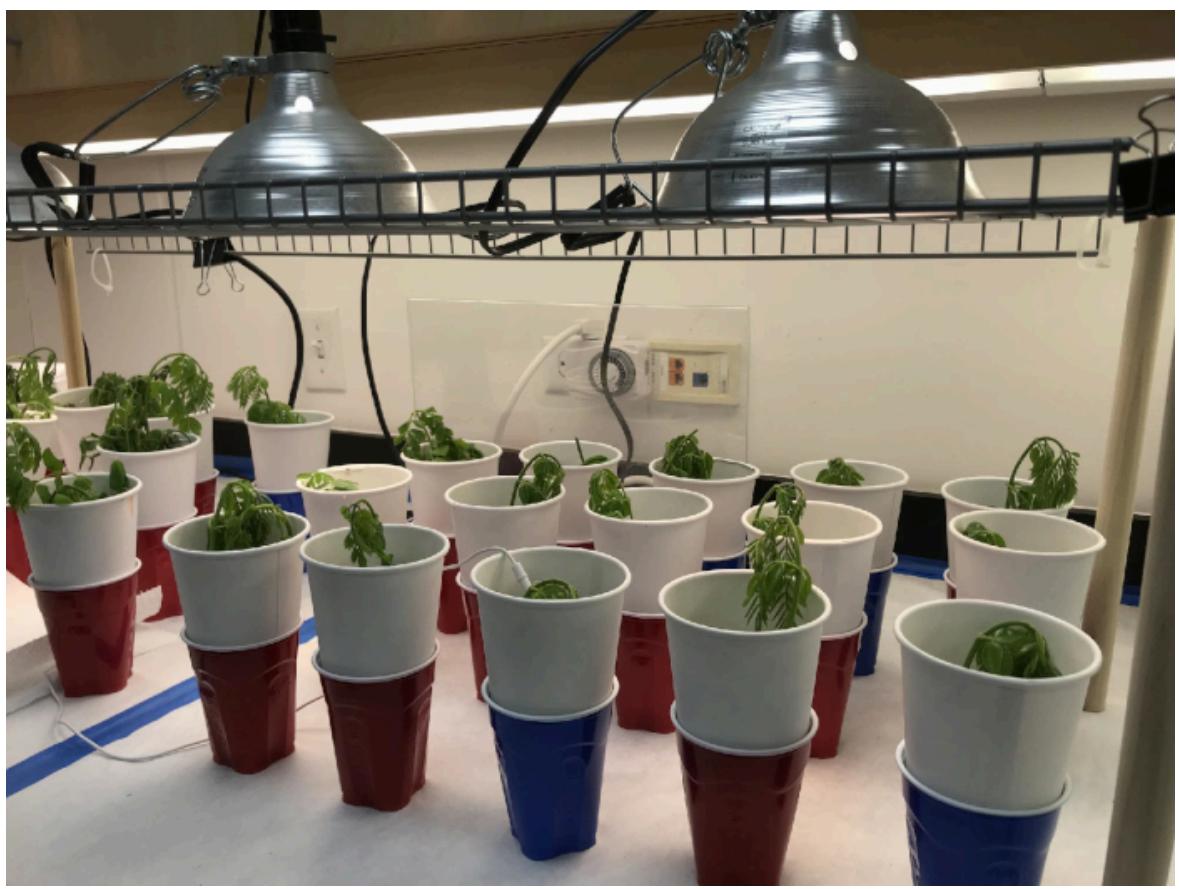

Supplementary Figure 6: Laboratory setup for larval care and maintenance. (A) Two cup system with each containing fresh terminal host material and larvae. (B) Temperature in the cups is maintained between $25^{\circ} \mathrm{C}-28^{\circ} \mathrm{C}$ for optimal larval activity and development by overhead clamp lights with $40 \mathrm{~W}$ incandescent bulbs. (C) A traceable memory monitoring thermometer with the temperature sensor placed directly in a cup is used to monitor temperature. Please click here to view a larger version of this figure.

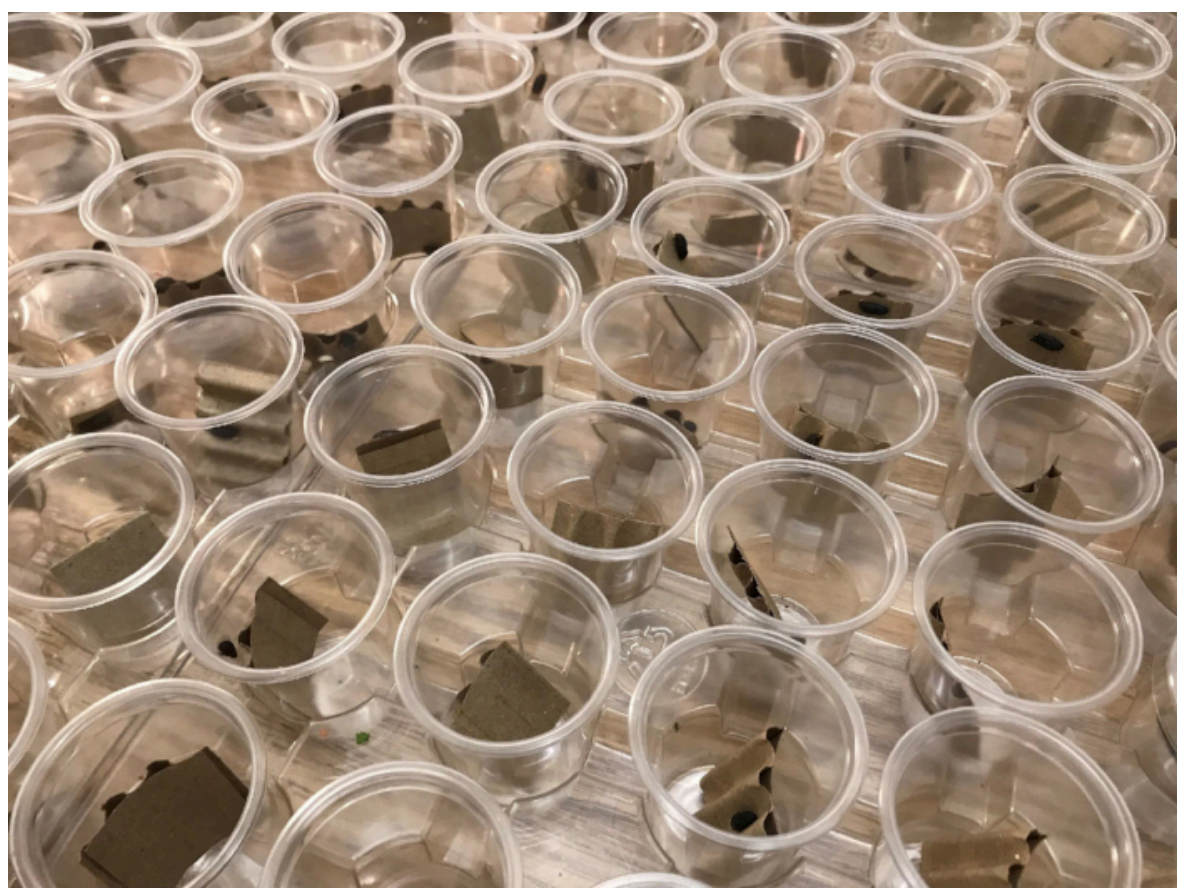

Supplementary Figure 7: Prepared pupation chambers. (A) Individual plastic portion cups housed on the clear plastic cup trays. (B) A corrugated paper square is placed in each plastic portion cup. (C) A single mature larva will be placed in each prepared plastic portion cup to pupate. Please click here to view a larger version of this figure. 


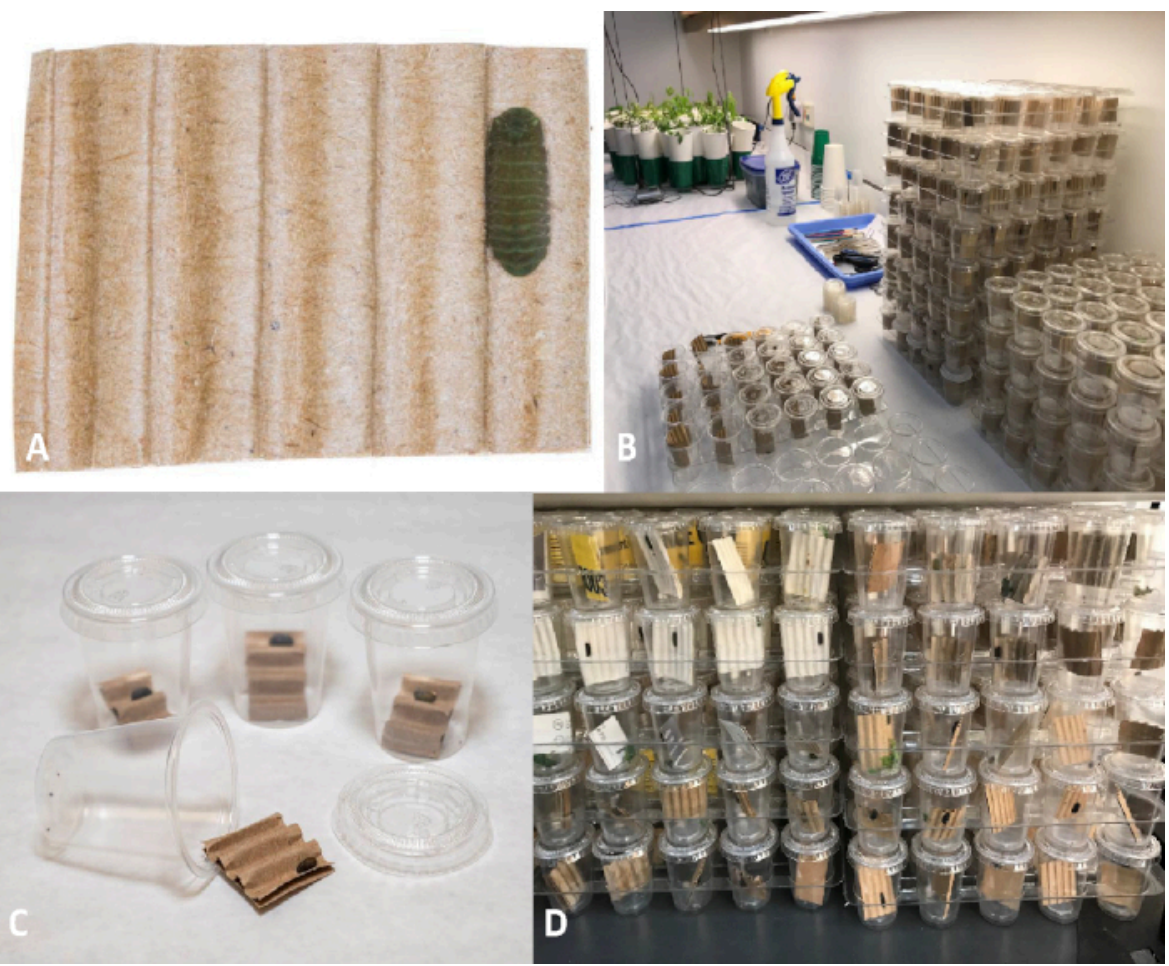

Supplementary Figure 8: Preparing larvae for pupation and pupal maintenance. (A) Mature larva ready to pupate on corrugated paper. It is a uniform dull greenish-brown and has lost any chevrons. (B) Pupation chambers ready to receive mature larvae adjacent to cups with feeding larvae. All pupation chambers with lids house larvae that are preparing to pupate. (C) Pupation chambers with pupae. (D) Banks of pupation chambers with pupae organized by date and maintained under laboratory conditions. Please click here to view a larger version of this figure.

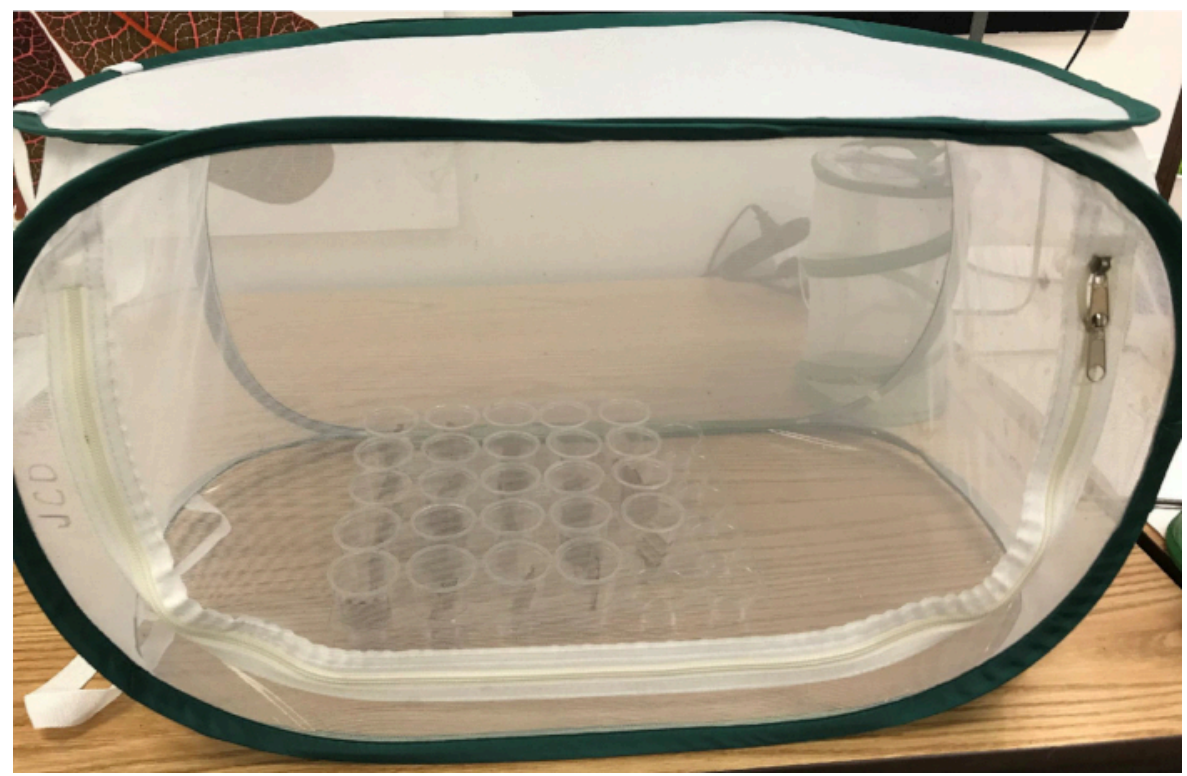

Supplementary Figure 9: Laboratory emergence cage. (A) A collapsible mesh pop-up rearing cage housing the occupied pupation chambers. (B) The lids of all the pupation chambers are removed to facilitate successful adult eclosion. (C) All resulting viable adult butterflies will be released into the screened flight cage to secure successful copulation. Please click here to view a larger version of this figure. 


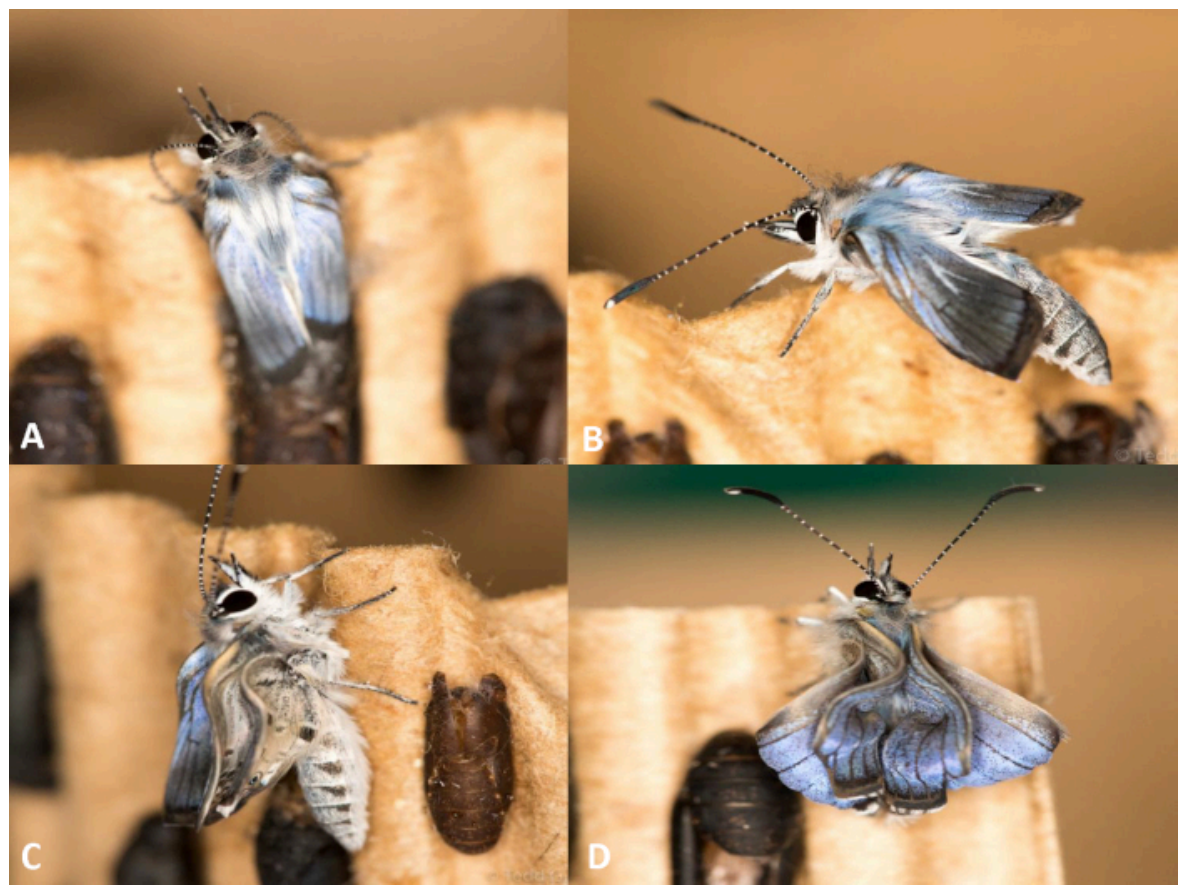

Supplementary Figure 10: Adult male butterfly successfully eclosing from pupa on a corrugated paper square. (A) Adult eclosing from pupa. (B) Adult fully removed from the pupal casing. (C) Adult positioned to expand its wings. (D) Adult expanding its wings. Please click here to view a larger version of this figure.

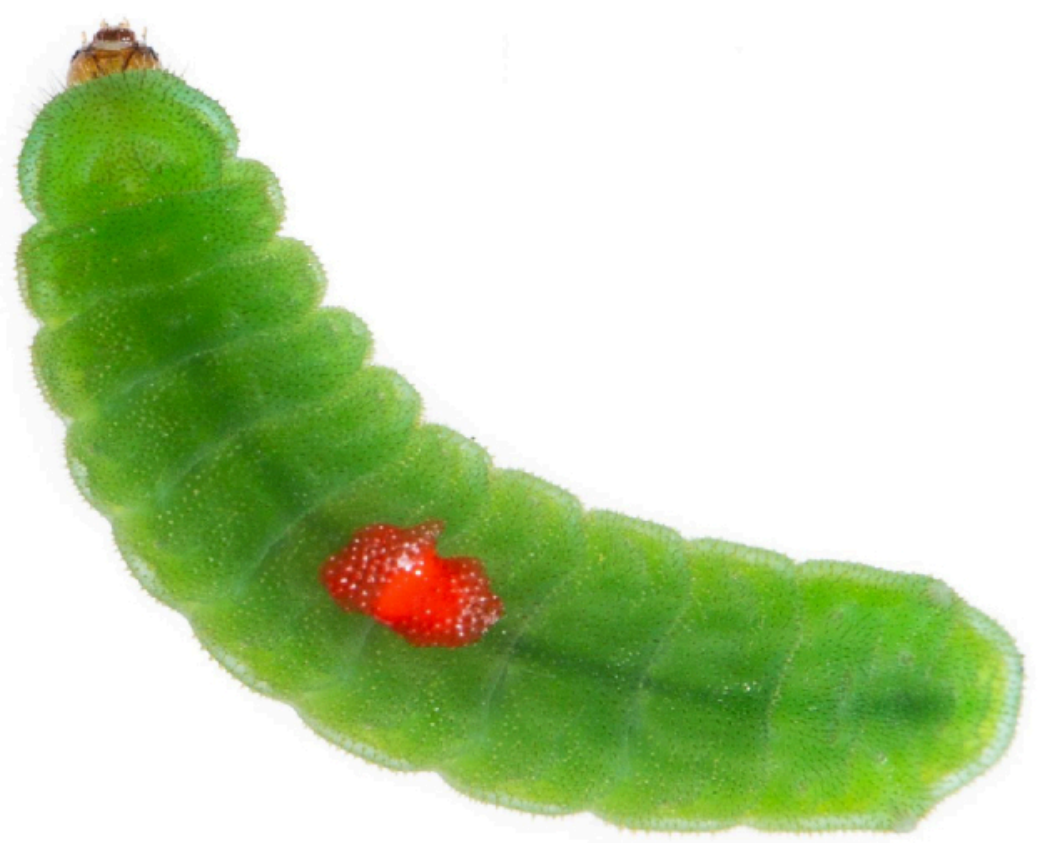

Supplementary Figure 11: Fifth instar larva marked with nontoxic luminous paint. (A) A small drop of contrasting red, nontoxic luminous paint is placed on the dorsum using a paintbrush to successfully mark the larva. Please click here to view a larger version of this figure. 


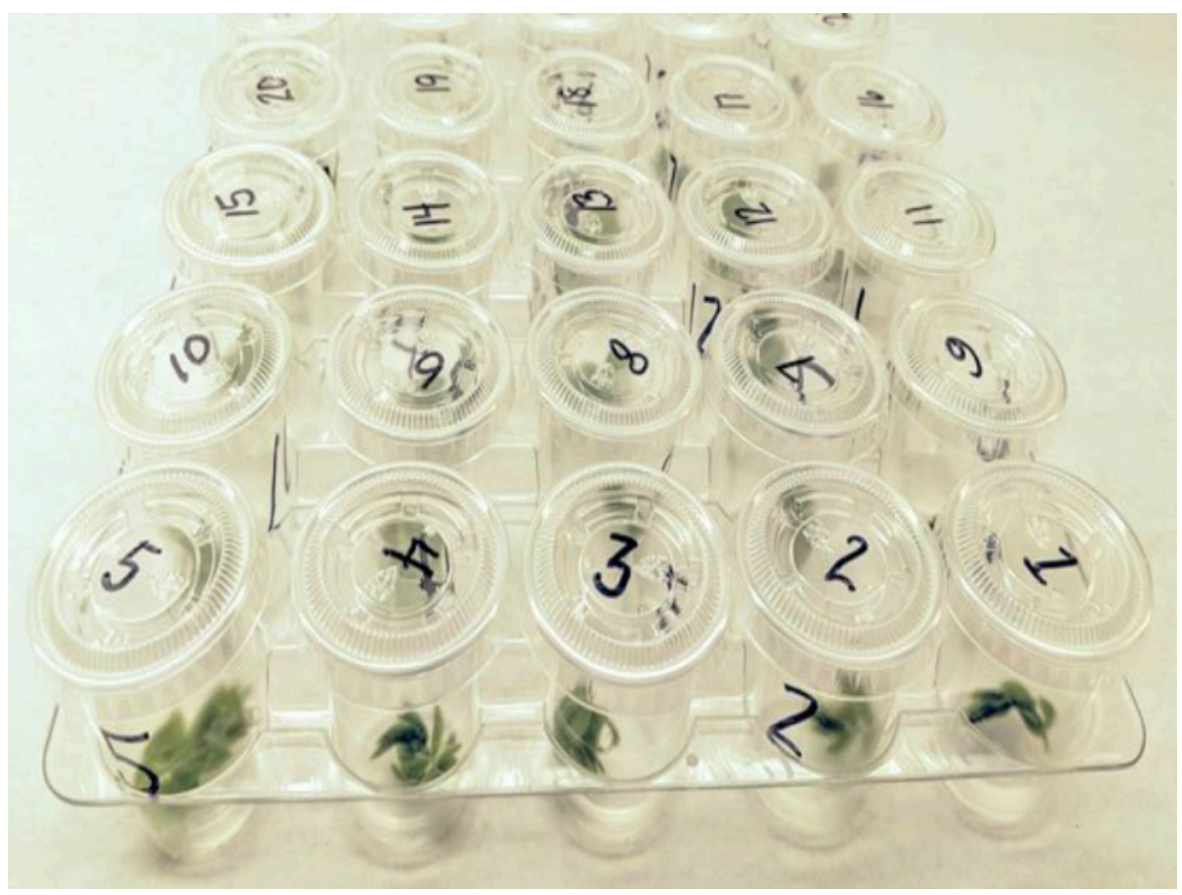

Supplementary Figure 12: Rearing set-up for life history study. (A) Uniquely labelled 2 ounce clear plastic portion cups. (B) A single larva is sequestered in each cup. (C) All larvae are individually tracked through all developmental stages from neonate to adult butterfly. Please click here to view a larger version of this figure.

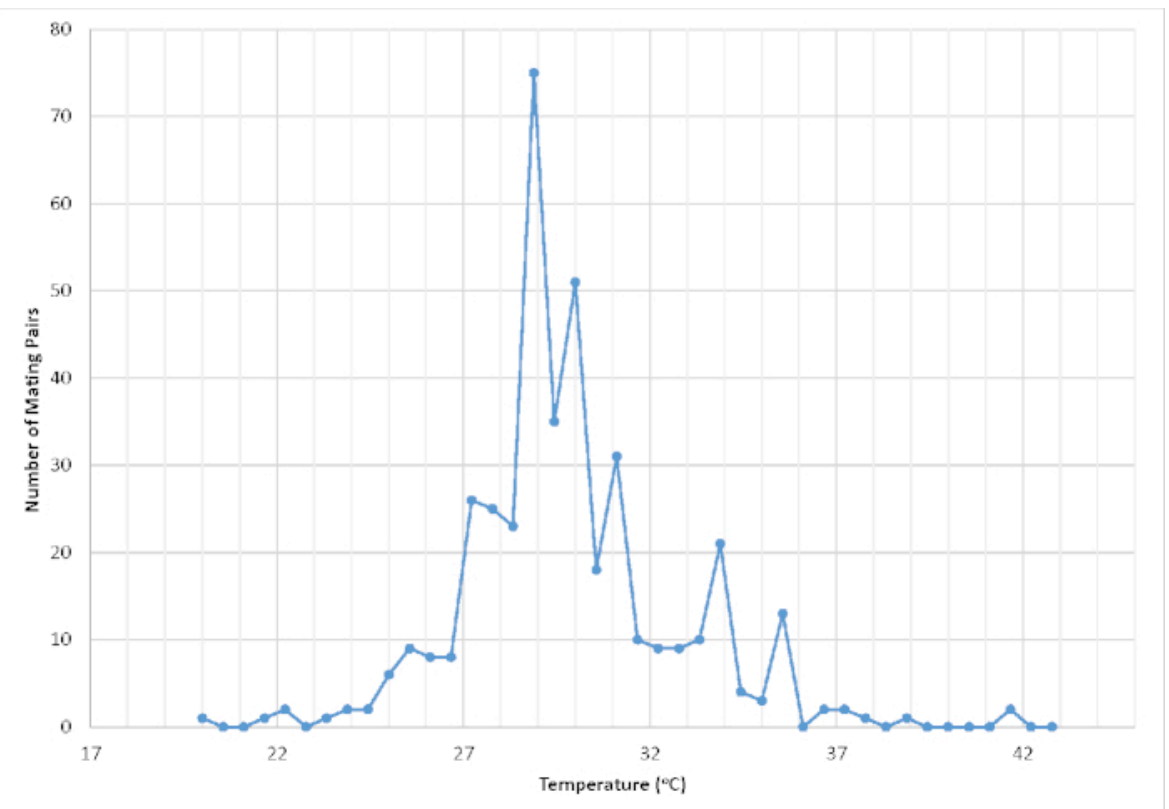

Figure 1: Number of recorded pairs in copula based on temperature $\left({ }^{\circ} \mathrm{C}\right)$ within a walk-in, screened flight cage housed in a

temperature-controlled greenhouse. The temperature was recorded within the first 2 min of a successful pairing event $(n=411)$. The resulting data were used to help refine the controlled environmental conditions in order to maximize mating success and ultimately overall captive propagation productivity. Please click here to view a larger version of this figure. 


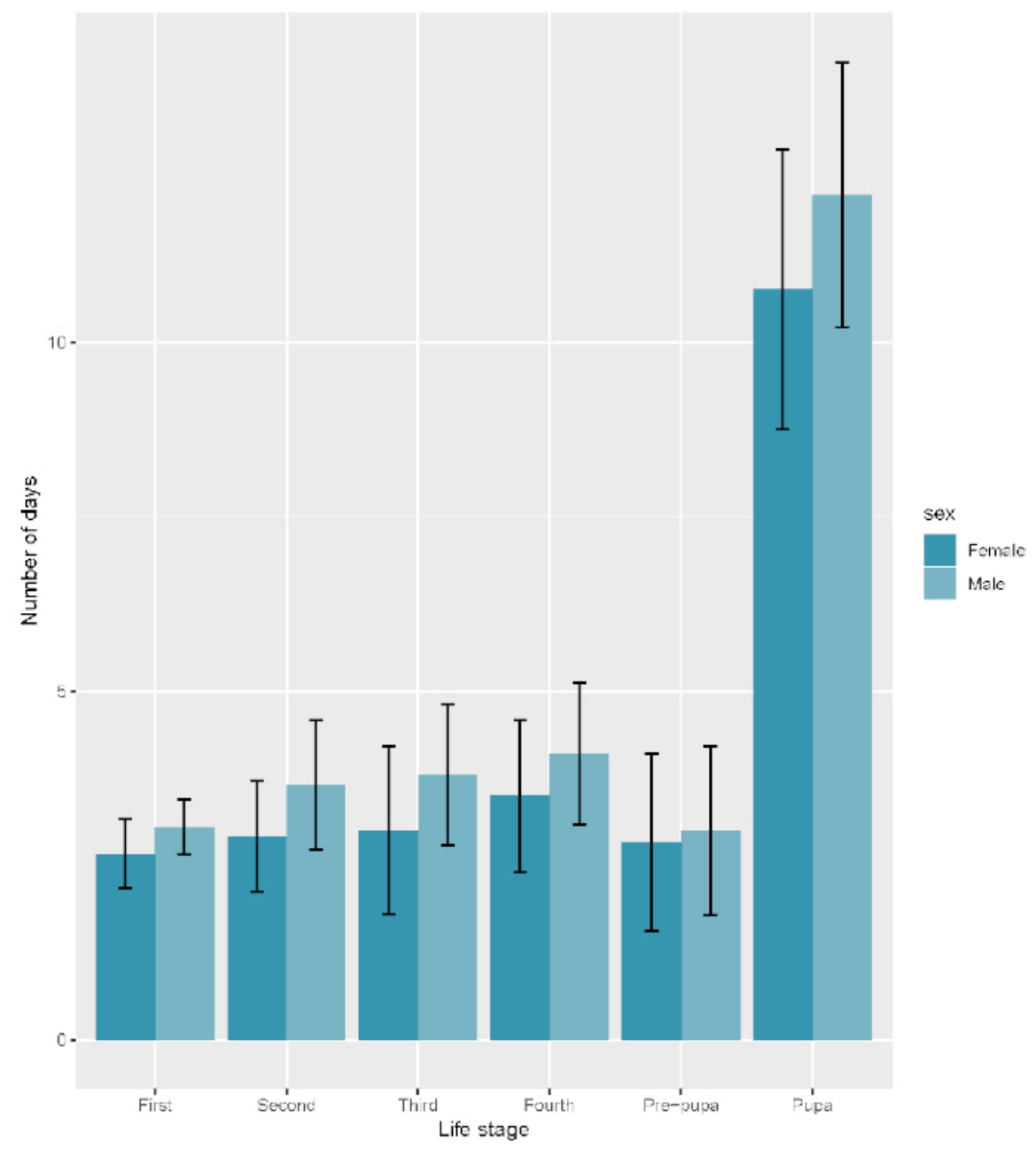

Figure 2: Mean development time (number of days) of each immature life stage. (A) Bars show the mean of each group, and error bars represent the upper and lower standard deviation values for each group. (B) Dark blue bars represent females, and light blue represent males. Please click here to view a larger version of this figure.

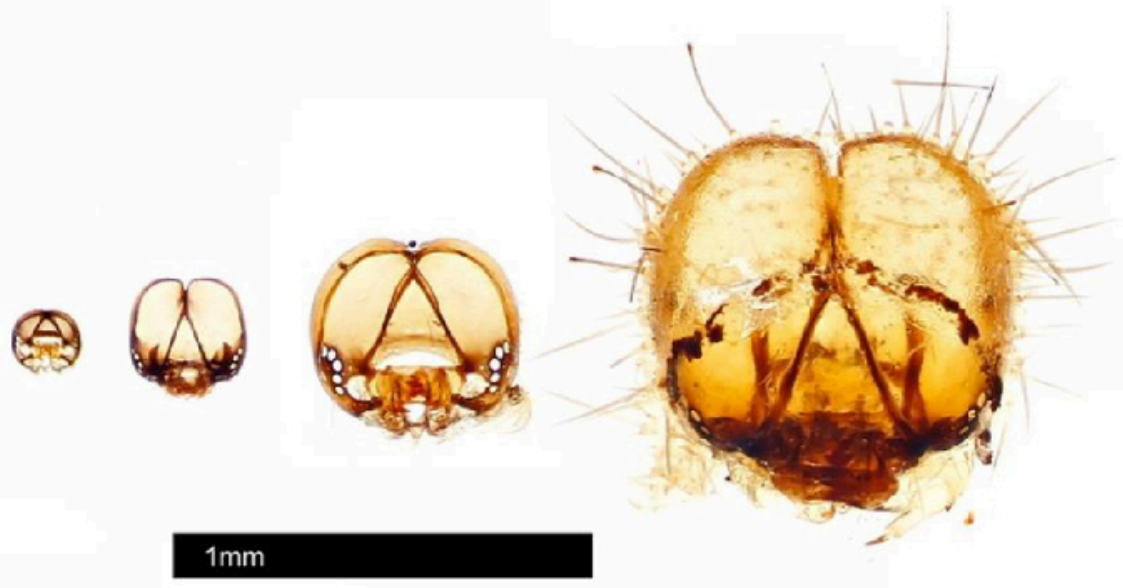

Figure 3: Head capsules collected from individual \#25 using life history protocol. Head capsules were photographed by Johnathan Bremer using an automontage system. Please click here to view a larger version of this figure. 


\begin{tabular}{|l|l|l|l|l|}
\hline Life stage & Mean body length $(\mathbf{m m})$ & Std. Error (length) & $\begin{array}{l}\text { Mean development time } \\
\text { (num. days) }\end{array}$ & Std. Error (dev. time) \\
\hline Instar I & $1.69478261(n=23)$ & 0.02152643 & $2.90625(n=32)$ & 0.08229783 \\
\hline Instar II & $2.77248958(n=32)$ & 0.04302826 & $3.375(n=32)$ & 0.16649857 \\
\hline Instar III & $5.45751042(n=32)$ & 0.12120829 & $3.5(n=32)$ & 0.20080483 \\
\hline Instar IV & $10.2369688(n=32)$ & 0.23653991 & $3.875(n=32)$ & 0.18917265 \\
\hline Instar V & $8.7625(n=2)$ & 2.6125 & $1.5(n=2)$ & 0.5 \\
\hline Instar VI & $10.2666667(n=1)$ & NA & $3(n=1)$ & NA \\
\hline Pre-pupa & $11.0858333(n=24)$ & 0.23948251 & $2.9375(n=32)$ & 0.21504641 \\
\hline Pupa & $9.0316129(n=31)$ & 0.12106792 & $11.6578947(n=38)$ & 0.3272288 \\
\hline
\end{tabular}

Table 1: Mean length and development time of each life stage. Standard error included for each variable, and sample size in parentheses.

\begin{tabular}{|l|l|l|}
\hline Life stage & Mean wing chord length $(\mathbf{m m})$ & Std. Error \\
\hline Adult & $12.63895(\mathrm{n}=38)$ & 0.1365516 \\
\hline Female & $12.960(\mathrm{n}=13)$ & 0.1465588 \\
\hline Male & $12.472(\mathrm{n}=25)$ & 0.1863205 \\
\hline
\end{tabular}

Table 2: Mean forewing wing chord length for adult butterflies. Includes means for females, males, and all adults (both sexes combined).

\begin{tabular}{|l|l|l|l|}
\hline LM Model 1 & Std. Error of estimate & t value & p-value \\
\hline Intercept & 1.9179 & 3.128 & $0.0046^{* *}$ \\
\hline Avg. length second instar & 0.6822 & -1.11 & 0.278 \\
\hline Avg. length third instar & 0.2928 & 0.476 & 0.6381 \\
\hline Avg. length fourth instar & 0.1373 & -0.57 & 0.5739 \\
\hline Avg. length pupae & 0.246 & 3.957 & $0.0005^{* * *}$ \\
\hline$* * * p<0.001 ;{ }^{* *} p<0.01 ;{ }^{*} p<0.05$. & & \\
\hline
\end{tabular}

Table 3: Coefficients table for linear regression model (LM Model 1) to evaluate relationship between average length of each life stage ( $n>30$ included in analysis) and adult wing chord length. Dependent variable: adult wing chord length $(\mathrm{mm})$.

\begin{tabular}{|l|l|l|l|}
\hline Coefficients & Std. Error of estimate & t value & $\operatorname{Pr}(>|\mathbf{t}|)$ \\
\hline Intercept & 1.7091 & 3.031 & $0.0053^{* *}$ \\
\hline Avg. length pupae & 0.1878 & 4.414 & $0.0002^{* * *}$ \\
\hline
\end{tabular}

Table 4: Stepwise regression (Stepwise 1). Dependent variable: adult wing chord length $(\mathrm{mm})$.

\begin{tabular}{|l|l|l|l|}
\hline LM Model 2 & Std. Error of estimate & t value & p-value \\
\hline Intercept & 1.1888 & 12.643 & $4.21 \mathrm{e}-12^{* * *}$ \\
\hline Num. days first instar & 0.3486 & 0.937 & 0.3583 \\
\hline Num. days second instar & 0.2603 & -0.686 & 0.4993 \\
\hline Num. days third instar & 0.2281 & 1.028 & 0.3141 \\
\hline Num. days fourth instar & 0.2048 & 2.378 & $0.0257^{*}$ \\
\hline Num. days pre-pupae & 0.222 & 1.133 & 0.2686 \\
\hline Num. days pupae & 0.2495 & 0.616 & 0.5435 \\
\hline Total num. days & 0.1913 & -1.454 & 0.1589 \\
\hline${ }^{* * *} p<0.001 ;{ }^{* *} p<0.01 ;{ }^{*} p<0.05$. & & \\
\hline
\end{tabular}

Table 5: Coefficients table for linear regression model (LM Model 2) to evaluate relationship between development time and adult wing chord length. Dependent variable: adult wing chord length $(\mathrm{mm})$. 


\begin{tabular}{|l|l|l|l|}
\hline Coefficients & Std. Error of estimate & t value & p-value \\
\hline Intercept & 0.89304 & 16.314 & $7.86 \mathrm{e}^{-16^{* * *}}$ \\
\hline Num. days second instar & 0.17974 & -1.809 & $0.0811^{\circ}$ \\
\hline Num. days fourth instar & 0.16917 & 2.075 & $0.0473^{*}$ \\
\hline Total num. days & 0.04184 & -1.787 & $0.0848^{\circ}$ \\
\hline${ }^{* * *} p<0.001 ;{ }^{* *} p<0.01 ;{ }^{*} p<0.05 ;$ & $p<0.1$ & & \\
\hline
\end{tabular}

Table 6: Stepwise regression (Stepwise 2) for development time. Dependent variable: adult wing chord length ( $\mathrm{mm}$ ).

\section{Discussion}

Here, we illustrate the effectiveness of this proven ex situ conservation breeding protocol for mass production of at-risk butterflies, and how it can be adapted for scientific research to help address key behavioral, life history, or ecological data gaps. Increased understanding of mean total development time (egg to adult), mean duration in each life stage, and optimal temperature for mating, for example, were used to help refine the protocol and enhance overall program success. The vast majority of existing protocols detail only organism husbandry methods and do not discuss data collection, scientific research, or the use of such results to help inform and potentially adapt ex situ methods.

This protocol requires daily organism husbandry. Organism health and productivity are maximized by clean rearing conditions, a lack of organism overcrowding, and the availability of high-quality larval host plant material. For the most part, we utilize disposable rearing supplies and containers (e.g., paper and plastic cups), and typically replace them regularly, often daily, and never reuse the material. This is both costeffective and minimizes the need for more labor-intensive sanitation of materials. Commonly used tools, however, such as entomological forceps, watercolor paint brushes, and small pop-up flight cages, as well as all rearing surfaces such as tabletops and laboratory bench tops are regularly sanitized using a $5 \%$ bleach solution. The exact schedule of sanitation is highly dependent on the frequency of use, organism phenology, and other variables, and should be tailored to the specific needs of each ex situ program. We additionally find that white butcher paper is useful to cover all rearing surfaces. It provides an inexpensive, easily deployable clean substrate, and the white background color facilitates sighting of any stray organisms. For daily husbandry, all laboratory personnel should always wear disposable laboratory exam gloves to minimize contamination and safeguard personnel from any potential skin irritation resulting from plant or organism handling. This is particularly critical if any laboratory personnel have household pets that require topical flea treatments. Even a small amount of active ingredient residue can be hazardous to captive livestock.

Additionally, care should be taken to minimize organism overcrowding. Overcrowding of larvae can quickly lead to reduced organism health and even cannibalism in certain taxa, particularly Lycaenidae. Regularly separating larvae to reduce numbers within rearing containers and/ or even isolating individual larvae as described in the life history portion of the protocol may be necessary. The ideal numbers per container may vary considerably based on the particular taxon and various ex situ program constraints such as available budget, laboratory facilities, and total number of husbandry personnel. We similarly recommend leaving adequate space between cups housing larvae to minimize the potential of organism movement between containers. Lastly, for larger captive populations, it is strongly recommended to separate stock between one or more laboratory facilities. This safeguarding strategy can help minimize catastrophic loss of the entire population due to disease or other unforeseen impacts.

Larval host plant quality and availability drives livestock production and strongly influences both larval development rates and overall population health. Nonetheless, few published reports or studies highlight this backstage requirement or discuss best nursery practices. Successful ex situ program planning must account for adequate plant quantities, production, and maintenance. As many larvae also require or prefer certain plant parts (e.g., terminal new growth, flower buds and inflorescences, fruit, etc.), effective staging to ensure appropriate plant phenology is required.

Additional considerations include appropriate demographic and genetic management, and the minimization of any potential negative effects of captivity. We recommend the development of a genetic management plan. This may include strategies to include the infusion of new genetic material on regular basis, maximize diversity and prevent close inbreeding, periodically evaluate key organism fitness variables, and monitor genetics at some level to enable comparison to extant populations and check captive stock health. Periodic comparison of the characteristics of captive individuals to individuals from the founding populations is also warranted $^{34,35}$.

These protocols represent proven best practices. They should be beneficial to a variety of researchers and conservation practitioners that can directly apply or adapt our methods to their own studies and ex situ at-risk butterfly or insect conservation and recovery programs. The specific outlined captive breeding protocol is likely most applicable to programs focused on other Lycaenidae, related taxa, or smaller sized taxa. Nonetheless, numerous components such as those involving securing successful courtship and copulation, adult maintenance with artificial nectar, maximizing oviposition, and general larval care could arguably be more broadly applied or adapted to a wider array of taxa. As mentioned earlier, while protocol flexibility should be emphasized, access to other established methodologies can help provide valuable insight and a viable departure point for adaptation and innovation. The methods presented for assessing various life history characteristics such as larval development time and the number of larval stadia arguably has broad applicability to other conservation breeding programs and at-risk taxa. We encourage others to help address key ecological data gaps when possible and to publish vetted protocols and program outcomes.

\section{Disclosures}

The authors have nothing to disclose. 


\section{Acknowledgments}

This work was supported by grants from the U.S. Fish and Wildlife Service's Conservation Recovery Initiative (F17AP00467) and the Disney Conservation Fund. Additional support was provided by the Florida Museum of Natural History and the Department of Entomology and Nematology at the University of Florida.

\section{References}

1. Thomas, J. A. Butterfly communities under threat. Science. 352 (6296), 216-218 (2016).

2. Swengel, S. R., Schlicht, D., Olsen, F., Swengel, A. B. Declines of prairie butterflies in the Midwestern USA. Journal of Insect Conservation. 15 (1-2), 327-339 (2011).

3. Habel, J. C. et al. Butterfly community shifts over two centuries. Conservation Biology. 30 (4), 754-762 (2016).

4. Gilburn, A. S. et al. Are neonicotinoid insecticides driving declines of widespread butterflies? Peer J. 3, e1402 (2015).

5. Sánchez-Bayo, F., Wyckhuys, K. A. G. Worldwide decline of the entomofauna: A review of its drivers. Biological Conservation. 232, 8-27 (2019).

6. Daniels, J. C., Magdich, M., Tolson, P. Butterfly recovery planning: Determining how to contribute. In Butterfly Conservation in North America: Efforts to Help Save Our Charismatic Microfauna. Edited by Daniels, J. C., 1-21, Springer Science+Business Media B.V., New York (2015).

7. U.S. Fish and Wildlife Service. Environmental Conservation Online System. Listed Animals. https://ecos.fws.gov/ecp/. July, (2019).

8. Schultz, C. B., Russell, C., Wynn, L. Restoration, reintroduction and captive propagation efforts for at-risk butterflies: a review. Israel Journal of Ecology and Evolution. 54, 41-61 (2008).

9. Grow, S., Allard, R., Luke, D. The role of AZA-accredited zoos and aquariums in butterfly conservation. In Butterfly Conservation in North America: Efforts to Help Save Our Charismatic Microfauna. Edited by Daniels, J. C., 23-34, Springer Science+Business Media B.V., New York (2015).

10. Crone, E. E., Pickering, D., Schultz, C. B. Can captive rearing promote recovery of endangered butterflies? An assessment in the face of uncertainty. Biological Conservation. 139, 103-112 (2007).

11. Sanchez, S. J., Daniels, J. C. The butterfly conservation initiative: Developing a new conservation vision through compound eyes. News of the Lepidopterists' Society. 49 (3), 75-77. (2007).

12. Wardlaw, J. C., Elmes, G. W., Thomas, J. A. Techniques for studying Maculinea butterflies: I. Rearing Maculinea caterpillars with Myrmica ants in the laboratory. Journal of Insect Conservation. 2 (1), 79-84 (1998).

13. Mattooni, R., Longcore, T., Krenova, Z., Lipman, A. Mass rearing the endangered Palos Verdes blue butterfly (Glaucopsyche lygdamus palosverdesensis:Lycaenidae). Journal of Research on the Lepidoptera. 37, 55-67 (1998).

14. Pearce-Kelly, P. et al. The captive rearing of threatened Orthoptera: a comparison of the conservation potential and practical considerations of two species' breeding programmes at the Zoological Society of London. Journal of Insect Conservation. 2 (3-4), 201-210 (1998).

15. Wells, C. N., Edwards, L., Hawkins, R., Smith, L., Tonkyn, D. A rearing method for Agrynnis (Speyeria) diana (Lepidoptera: Nymphalidae) that avoids diapause. Psyche. 2011, Article ID 940280, 1-6. (2011).

16. Grosboll, D. N. Captive Rearing the Endangered Mardon Skipper (Polites mardon) and Taylor's Checkerspot (Euphydryas editha taylori) Butterflies: Initial Results (Lepidoptera, Nymphalidae). Proceedings of the species at risk, pathways to recovery conference., Victoria, 2004. Species at Risk Pathways to Recovery Conference Organizing Committee, Victoria, 1-6 March (2004).

17. Barclay, E., Arnold, M., Andersen, M., Shepherdson, D. Husbandry manual: Taylor's checkerspot (Euphydryas editha taylori.). September 2009, 1st edition. Oregon Zoo, Portland, Oregon (2009).

18. Johnson, J. et al. Captive Rearing of the Laguna Mountains Skipper (Pyrgus ruralis laguanae.). Final Report, Cooperative Agreement No. 814307J024. (2010).

19. Linders, M. Captive rearing and translocation of Taylor's checkerspot in South Puget Sound: 2011-2012. 2012 Annual Progress Report to the ACUB Technical Review Committee. (2012).

20. Linders, M., Lewis, K. Captive rearing and translocation of Taylor's checkerspot butterfly (Euphydryas editha taylori.): South Puget Sound, Washington, 2012-2013. 2013 Annual Report to the US Fish and Wildlife Service (Cooperative Agreement F12ACI00835), Joint Base LewisMcChord Fish and Wildlife Program and JBLM-ACUB Technical Review Committee. (2013).

21. Department of Conservation and Research, Toledo Zoo. Propagation Handbook for the Karner Blue Butterfly Lycaeides melissa samuelis. Fourth edition. (2006).

22. Johnson, J. J. et al. Captive Rearing of Lange's Metalmark Butterfly, 2011-2015. 2016 United States Fish and Wildlife Service, CVPIA Habitat Restoration Program (F11AP00168). (2016).

23. Andersen, M.J. et al. Oregon Silverspot Butterfly Husbandry Manual. Oregon Zoo, Portland, Oregon. (2010).

24. Washington Department of Fish and Wildlife. Threatened and Endangered Wildlife in Washington: 2012 Annual Report. Listing and Recovery Section, Wildlife Program, Washington Department of Fish and Wildlife, Olympia. (2013).

25. McGowan, P. J. K., Traylor-Holzer, K., Leus, K. IUCN guidelines for determining how ex situ management should be used in species conservation. Conservation Letters. 10 (3), 361-366 (2017).

26. Pearce-Kelly, P. et al. The conservation value of insect breeding programmes: Rationale, evaluation tools and example programme case studies. In Insect Conservation Biology: Proceedings of the Royal Entomological Society's 23nd Symposium. Edited by Stuart, A. J. A, New, T. R., Lewis, O. T., 57-75, CABI (2007).

27. U.S. Fish and Wildlife Service. Policy Regarding Controlled Propagation of Species Listed Under the Endangered Species Act. United States Federal Register. 65 (183), 56916-56922 (2000).

28. IUCN/SSC. Guidelines on the use of ex situ management for species conservation. Version 2.0. IUCN Species Survival Commission. Gland, Switzerland (2014).

29. Sutherland, W. J., Pullin, A. S., Dolman, P. M., Knight, T. M. The need for evidence-based conservation. Trends in Ecology \& Evolution. 19 (6), 305-308 (2004).

30. Daniels, J. C., Nordmeyer, C., Runquist, E. Improving standards for at-risk butterfly translocations. Diversity., 10, 67. (2018). 
31. Saarinen, E. V. Population genetics of the endangered Miami blue butterfly Cyclargus thomasi bethunebakeri.: implications for conservation. Ph.D. Dissertation, University of Florida, Gainesville (2009).

32. Becker, T. Propagation and repatriation of the regal fritillary butterfly. http://titag.org/2016/2016papers/beckerregal.pdf. July, (2019).

33. R Core Team. R A Language and Environment for Statistical Computing. R Foundation for Statistical Computing. Vienna, Austria (2016).

34. Schultz, C. B., Dzurisin, J. D., Russell, C. Captive rearing of Puget blue butterflies (Icaricia icarioides blackmorei) and implications for conservation. Journal of Insect Conservation. 13 (3), 309-313 (2009).

35. Frankham, R., Loebel, D. A. Modeling problems in conservation genetics using captive Drosophila populations: Rapid genetic adaptation to captivity. Zoo Biology. 11 (5), 333-342 (1992). 